\title{
User-Assisted Store Recycling for Dynamic Task Graph Schedulers
}

\author{
MEHMET CAN KURT, Quantcast Corporation \\ SRIRAM KRISHNAMOORTHY, Pacific Northwest National Laboratory \\ GAGAN AGRAWAL, The Ohio State University \\ BIN REN, The College of William and Mary
}

\begin{abstract}
The emergence of the multi-core era has led to increased interest in designing effective yet practical parallel programming models. Models based on task graphs that operate on single-assignment data are attractive in several ways. Notably, they can support dynamic applications and precisely represent the available concurrency. However, for efficient execution, they also require nuanced algorithms for scheduling and memory management. In this article, we consider memory-efficient dynamic scheduling of task graphs. Specifically, we present a novel approach for dynamically recycling the memory locations assigned to data items as they are produced by tasks. We develop algorithms to identify memory-efficient store recycling functions by systematically evaluating the validity of a set of user-provided or automatically generated alternatives. Because recycling functions can be input data-dependent, we have also developed support for continued correct execution of a task graph in the presence of a potentially incorrect store recycling function.

Experimental evaluation demonstrates that this approach to automatic store recycling incurs little to no overheads, achieves memory usage comparable to the best manually derived solutions, often produces recycling functions valid across problem sizes and input parameters, and efficiently recovers from an incorrect choice of store recycling functions.
\end{abstract}

\section{CCS Concepts: • Computing methodologies $\rightarrow$ Parallel programming languages; $\bullet$ Theory of computation $\rightarrow$ Parallel computing models;}

Additional Key Words and Phrases: Task parallelism, memory management, dynamic scheduling

ACM Reference Format:

Mehmet Can Kurt, Sriram Krishnamoorthy, Gagan Agrawal, and Bin Ren. 2016. User-assisted store recycling for dynamic task graph schedulers. ACM Trans. Archit. Code Optim. 13, 4, Article 55 (December 2016 ), 24 pages.

DOI: http://dx.doi.org/10.1145/3018111

\section{INTRODUCTION}

The increase in hardware parallelism exposed by multi- and many-core systems has exacerbated the programmer effort required to manually map a given program to the hardware processing units in a load-balanced manner. Data-flow- or task-graph-based

This work was supported in part by the U.S. Department of Energy's (DOE) Office of Science, Office of Advanced Scientific Computing Research, under award 63823. Pacific Northwest National Laboratory is operated by Battelle for DOE under Contract DE-AC05-76RL01830. This work was also partially supported by NSF awards CCF-1319420 and CCF-1629392 and the Department of Energy (DOE) award DE-SC0014135 to The Ohio State University.

Authors' addresses: M. C. Kurt, Quantcast Corporation, 795 Folsom St, San Francisco, CA 94103; email: mcankurt@quantcast.com; G. Agrawal, The Ohio State University, 395 Dreese Laboratories, 2015 Neil Avenue Columbus, OH 43210; email: agrawal@cse.ohio-state.edu; S. Krishnamoorthy, Pacific Northwest National Laboratory, 902 Battelle Blvd, Richland, WA 99354; email: sriram@pnnl.gov; B. Ren, The College of William and Mary, McGlothlin-Street Hall 126, 251 Jamestown Rd., Williamsburg, VA 23185; email: bren@cs.wm.edu.

Permission to make digital or hard copies of part or all of this work for personal or classroom use is granted without fee provided that copies are not made or distributed for profit or commercial advantage and that copies show this notice on the first page or initial screen of a display along with the full citation. Copyrights for components of this work owned by others than ACM must be honored. Abstracting with credit is permitted. To copy otherwise, to republish, to post on servers, to redistribute to lists, or to use any component of this work in other works requires prior specific permission and/or a fee. Permissions may be requested from Publications Dept., ACM, Inc., 2 Penn Plaza, Suite 701, New York, NY 10121-0701 USA, fax +1 (212) 869-0481, or permissions@acm.org.

(c) 2016 ACM 1544-3566/2016/12-ART55 $\$ 15.00$

DOI: http://dx.doi.org/10.1145/3018111 
programming systems, which have existed for the past four decades [Arvind 1981; Dennis 1974; Najjar et al. 1999], expose significantly more concurrency than the available hardware parallelism, allowing for automated balanced mapping of work among processor cores. In recent years, there has been renewed interest in this broad class of programming models [Budimlić et al. 2010; Duran et al. 2011; Matsakis 2012; Agrawal et al. 2010]. However, making these models flexible while also enabling timeand space-efficient implementations continues to be an open challenge.

This article focuses on memory-efficient execution of programs structured as task graphs and processed by a dynamic scheduler. To motivate the problem, consider a data-flow (or single-assignment) task graph. The edges in such a task graph represent only the true dependences as ordering constraints. Each task in these task graphs produces a unique data item, which is not subsequently modified by other tasks. This representation maximally exposes concurrency in the program and enables the most effective use of available hardware parallelism. However, naively scheduling such task graphs can quickly overrun the memory available in a parallel system. Given that the definition-use relationships in a dynamically executing task graph are not known a priori, any specified data item might have a potential future use. Thus, it cannot be garbage collected until the task graph completes execution. Other approaches to optimize memory usage often require additional information about the number of uses or the last use of a given data item [Kurt et al. 2014; Sbirlea et al. 2012]. Also, many approaches to addressing the memory issue require knowledge of the static task graph [Schnorf et al. 1993; Sbirlea et al. 2014; Gérard et al. 2012], a requirement that can limit the attractiveness of the task model itself.

It should be noted that several different task-based programming models exist, and our work is applicable to only a subset of these. Systems such as Nabbit [Agrawal et al. 2010], QUARK [YarKhan et al. 2011], StarPU [Augonnet et al. 2011], OmpSs [Duran et al. 2011], and XKaapi [Gautier et al. 2013] express data-flow dependences without building on the static single-assignment form. They require users to manage memory while specifying inter-task dependences. We specifically focus on programming models that schedule task graphs in single-assignment form, similar to Concurrent Collections (CnC) [Budimlić et al. 2010]. These models simplify programming by not requiring users to worry about memory limits, instead necessitating effective automated approaches to memory management.

In general, several approaches exist to manage memory when the tasks in a task graph are explicitly enumerated. In this case, schedules can be constructed for execution of tasks, memory allocation, and deallocation. Often, the task graphs in many applications are compactly specified with the specification being much smaller in size than the number of tasks. We consider the problem of memory-efficient scheduling of compactly specified task graphs by exploiting the structure of the data-flow relationships. In particular, rather than derive a memory allocation/deallocation schedule for each task executed, we attempt to derive such a schedule for each task type in the compact specification.

In this article, we present a different approach for optimizing memory usage when scheduling data flow of single-assignment task graphs. We posit that the additional information required to support memory-efficient execution either requires significant additional effort from the programmer or is simply not available. In fact, a study [Budimlic et al. 2008] to automate extraction of use counts in Intel CnC observes that determining correct use counts can be a significant challenge for programmers. Thus, to further improve the functionality and programmability of these types of programming models, we address the problem of memory minimization when the task graph is generated dynamically, and information about last use (or the number of uses) of a memory block is not available. 
Specifically, we present an approach to recycling the store across tasks in a task graph. Recycling operations during execution are dictated by functions that we refer to as store recycling functions. We address a number of issues related to realizing a recycling-based task graph execution. As a first step toward simplifying the programmer's task, we present an algorithm to verify that a given store recycling function is correct (e.g., the recycling does not cause any overwrites to live data or lead to a data race). For the case of demand-driven schedulers, i.e., schedulers that execute task graphs starting from the sink vertex and transitively executing the tasks it depends on, we have developed mechanisms for such detection to be carried out with low overheads during program execution and that enable recovery from incorrect overwrites within the same run. Thus, the programmer can execute an application with a potentially incorrect recycling function and still obtain a correct execution without a large slowdown.

To further improve programmability, we present an approach toward automatically deriving a correct recycling function. Specifically, we consider compositions of incoming dependences as potentially valid recycling functions, determine which of them is correct, assess the reuse facilitated by correct functions, and choose the one that leads to the largest number of recycled stores. The correctness determination can be done via execution on a smaller problem size. While this does not ensure correctness of recycling function on a different input data set (as dependences can be input-dependent), recall that we can verify the correctness with low overheads during a production run.

Experimental evaluation demonstrates the usefulness of this novel approach to managing memory when dynamically scheduling task graphs. We demonstrate that the cost of identifying valid store recycling functions is low, the automatically derived recycling functions achieve the same performance and memory usage as hand-optimized versions and are effective across problem sizes, and invalid recycling functions can be efficiently identified and recovered from.

To summarize, the primary contributions of the article are:

-An approach to memory-efficient task graph scheduling using the novel notion of store recycling functions.

-Definition of the characteristics of correct and efficient store recycling functions.

-Representation of a collection of feasible store recycling functions as a composition of true dependences.

-An algorithm to efficiently evaluate correctness of a given, or set of given, user-defined or automatically derived store recycling functions.

-An algorithm to dynamically recover from an incorrect choice of store recycling functions.

\section{TASK GRAPH SCHEDULING}

A task graph is a directed acyclic graph consisting of nodes that denote tasks and edges that designate inter-task dependences. An edge from task A to task B indicates that task B cannot begin execution until task $\mathrm{A}$ has completed execution. Task $\mathrm{A}$ is said to be a (dependence) predecessor of task $\mathrm{B}$, whereas task $\mathrm{B}$ is a (dependence) successor of task A. Certain task graph models, such as $\mathrm{CnC}$ [Budimlić et al. 2010], employ alternative representations with explicit tasks and data items, where tasks produce data items once the data items they depend on are ready. However, to simplify the discussion, we will employ a representation of task graphs consisting only of tasks. In particular, each task is assumed to produce one data item, allowing us to treat a data item and the task that produces it as being synonymous. Task graphs in which tasks produce multiple data items can be handled by introducing additional tasks representing the data items. For example, consider a task $t 1$ producing data items $a$ and $b$, which are consumed by tasks $t 1 a$ and $t 1 b$, respectively. We model this task using two tasks, $t 1$ and $t 1^{\prime}$, where $t 1$ "holds" the memory associated with the data item $a$ while $t 1^{\prime}-$ a task 
with no actual work-holds the memory associated with data item $b$. The edges in this task graph are given by the pairs $\left(t 1, t 1^{\prime}\right),(t 1, t 1 a)$, and $\left(t 1^{\prime}, t 1 b\right)$. Task graph models typically distinguish between intermediate data items and those exposed to the outside environment, also referred to as the output data items. The output data items cannot obviously be deallocated during task graph execution, but efficient execution requires design of memory reclamation strategies for the intermediate data items.

Various memory management strategies have been studied in the literature for these intermediate data items. In the first group of approaches, task graph schedulers are agnostic of data items and leave the burden of memory management to the user. When using these schedulers, the user code explicitly maps the outputs of multiple tasks to the same memory region. This, however, is a tedious and error-prone process. There is potential for incorrectness because of untimely memory overwrites or inefficiency stemming from ordering constraints introduced when tasks use the same memory region.

As the next group, reference counting garbage collectors and those in managed runtime systems (e.g., mark-and-sweep collection schemes), declare an object with no references from live variables as dead and ready to be deallocated. However, during dynamic task graph execution, all data items can be accessed by any pending tasks. Therefore, no tasks can be garbage collected until task graph execution completes.

Another approach to memory management requires users to specify the number of uses or last use for each data item. This information is independent of the schedule and can enable eager memory reclamation [Budimlić et al. 2010; Budimlic et al. 2008]. Unfortunately, providing this information imposes an additional burden on the programmer, i.e., analyzing the task graph to compute the exact number of uses for each data item (which likely will differ as the problem size changes). Moreover, the exact number of uses on each data item is not always available beforehand. The complete set of consumers of a data item might dynamically change across different runs, depending on factors such as input from the environment, conditional statements in task's computations, and dynamic generation of new tasks. One way to determine reference counts in these conditions is to expand and analyze the task graph completely before actual execution. However, such analysis, which should be repeated for each different problem instance or size, can be extremely expensive.

One alternative to the previous group of studies is to schedule task graphs under strict memory constraints [Sbirlea et al. 2014]. While this solution can be desirable in many cases, it is also extremely challenging to achieve for dynamic schedulers due to the possibility of resource utilization deadlock. In addition, applications typically run in environments with some flexibility in their memory usage. Thus, using the absolute minimum memory often is not necessary.

Because of the limitations of the preceding approaches, we are interested in designing an automated yet efficient approach to dynamically schedule task graphs in a memoryefficient fashion. As we explain in Section 3, our approach is based on recycling the store as the execution proceeds, which allows us to specify memory management constraints in terms of tasks in the task graph.

Demand-driven execution. Our approach involves two components: (a) determining and employing recycling functions and (b) recovering from the incorrect choice of recycling functions to ensure continued correct execution. Whereas our recycling approach attempts to identify recycling functions that are valid irrespective of the schedule, the recovery approach is specifically designed for demand-driven execution of task graphs. In the case of demand-driven schedulers, execution begins with the sink vertex, the task whose execution indicates complete execution of the task graph. Before a task can begin execution, all tasks it depends on are created and executed concurrently. In a task graph, multiple tasks might depend on the same upstream task. To avoid 


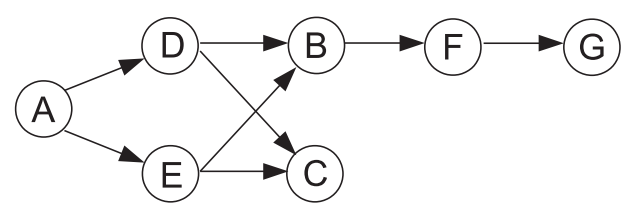

Fig. 1. A task graph example, where task $B$ can safely recycle the store assigned to task $A$ but not the one assigned to task $C$.

duplicated execution of such tasks by multiple threads, each task is associated with a unique key. The scheduling algorithm maximally exposes concurrency while ensuring efficient coordination among threads for correct and non-redundant execution. We refer the reader to Agrawal et al. [2010] for more detailed exposition of the demand-driven scheduler we employ for recovery.

Assumptions. We assume that all tasks produce, at most, one output data block. For simplicity, we consider task graphs where all data blocks are the same size. We focus on task graphs with task execution times much larger than task descriptor management times. Executing the tasks in a demand-driven fashion requires storage of task descriptors along the critical path, which can grow with task graph size. As typical in practice, we consider task graphs where the space to store the data produced by the tasks is far greater than the space to store the task descriptors. In demanddriven dynamic execution of a task graph, the data blocks accessed by a task are not known in advance. In particular, a task can access any of the data blocks produced by tasks it transitively depends on. However, large task graphs are typically constructed in a parametric form with similar data access relationships across problem sizes. In formulating our recycling strategy, we assume this similarity across problem sizes.

\section{OUR APPROACH: STORE RECYCLING FUNCTIONS}

Excluding input data, the task graph (shown in Figure 1) can be executed with space to store four data blocks, where each task produces one data block. This requires deallocating data blocks when they are no longer required. In general, achieving the optimal space bound requires a specific schedule. Without garbage collection, constraints on the schedule, or prior knowledge of the last use of each data block, all data blocks need to be retained until the task graph is completely executed. This increases the space requirement to seven data blocks. We are interested in an automated approach to garbage collection that attempts to determine the last use for the data block in a schedule-independent fashion.

A store recycling function maps each task $T$ in a directed acyclic graph to another task so that $T$ can reuse the memory segment containing the mapped task's output for its own output. For instance, a specification $\operatorname{Recycle}(B)=A$ states that the output of task $B$ occupies the same memory region as the output of task $A$. Here, we refer to task $A$ as a recycle predecessor of task $B$ and task $B$ as a recycle successor of task $A$.

The key technical issue is that several correctness and efficiency considerations can arise in choosing recycling function(s).

\subsection{Recycling Constraints}

A recycling operation dictated by a store recycling function is correct if and only if it complies with the causality relations among tasks and does not lead to a data race. For example, consider the store recycling function that maps task $B$ to task $A$ in the task graph in Figure 1. A schedule that employs such a store recycling function should order the execution of $A$ to precede the execution of $B$. A store recycling function is correct if and only if every valid schedule for the task graph satisfies this ordering constraint. In 
other words, any dependence induced by the store recycling function should already be implied by the edges in the task graph. In addition, there are correctness requirements to ensure that we do not have an early overwriting of task outputs. Specifically, store recycling should not cause a task's output to be overwritten before all of its uses are complete. Figure 1 shows that task $A$ has two immediate successors ( $D$ and $E$ ), which finish execution before $B$ starts. Hence, $B$ recycling $A$ 's output would not cause early overwriting of $A$ 's output. We formulate the aforementioned requirements and define the first constraint for a recycling operation to be correct as follows:

Constraint 1: If task $B$ recycles the output of task $A$, then $A$ and all of $A$ 's immediate successors must causally precede $B$.

Consider the concurrent tasks $B$ and $C$ in Figure 1. Although the recycling operation $\operatorname{Recycle}(B)=C$ violates the first constraint, injecting an additional dependence edge from $C$ to $B$ is potentially possible. However, introduction of such additional dependences can lead to cycles and, thus, deadlock. They also may incur performance penalties because the scalability achievable by task graph schedulers is fundamentally limited by the degree of parallelism available, or, alternatively, the computation's critical path length. Note that this constraint can be ensured for a recycling operation by imposing additional dependences on the task graph.

A store recycling function can potentially map two different tasks onto the same task. Consider the recycling operation $\operatorname{Recycle}(C)=A$ in addition to $\operatorname{Recycle}(B)=A$. Existing dependences in the task graph indicate that $A$ and $A$ 's immediate successors (again $D$ and $E$ ) causally precede both $B$ and $C$, satisfying the first constraint. Although these recycling operations are individually correct, they both cannot be applied because $B$ and $C$ can run concurrently and lead to a data race on $A$ 's output. To prevent such cases, we define the second constraint as follows:

Constraint 2: Two tasks $B$ and $C$ can recycle the same task $A$ 's store only if $B$ (or

$C$ ) can recycle the store associated with $C$ (or $B$ ).

As a correct recycling scenario exemplifying the second constraint, one can consider the recycling operations Recycle $(B)=A$ and $\operatorname{Recycle}(G)=A$. Because $G$ can potentially recycle $B$ 's output, these recycling operations can both be applied within the same run (the former followed by the latter).

Our approach is based on verification of a set of store recycling functions against the two recycling constraints presented previously. Ideally, a store recycling function that is employed during execution must satisfy these constraints for every recycling operation under any possible schedule and problem instance of the given task graph. However, due to the dynamic dependence relations and new task generations inherently present in dynamic task graphs, such a global verification cannot be performed without exhausting the space of possible configurations. Hence, we attack this problem using a two-phase process. As we explain in Section 4, we first prune the space of candidate store recycling functions by verifying that it leads to correct execution on a smaller representative instance of the task graph. Next, the list of verified recycling functions are fed into the second phase, where we execute the actual task graph instance with one of the recycling functions on the list. As detailed in Section 5, the second phase is able to detect any incorrect recycling operations and fix their side effects with low overheads.

\section{EVALUATING STORE RECYCLING FUNCTIONS}

In this section, we first describe our approach to evaluating a collection of user-specified store recycling functions in terms of correctness and efficiency then explain how to generate these functions automatically. 


\subsection{Checking for Correct Execution}

Consider the verification of a single recycling function. During verification, we mimic the actions of the recycling function without actually performing them. The actual task graph execution could be performed using a different or no recycling function. For ease of discussion, in the remainder of this section, we refer to the recycle operations being evaluated as if they are employed in the execution.

The two constraints in Section 3 imposed on a store recycling function relate to causality relationships between tasks. A simple scheme can execute the task graph and retain predecessor and successor information for all tasks, as well as verify all recycling operations upon termination. However, this could incur high overheads. Our idea is to perform on-the-fly checks, using vector clocks to concisely represent causality [Raychev et al. 2013]. Specifically, we associate a vector clock of length $N$ with each task, where $N$ is the number of tasks. Each element in a vector has a value of either 0 or 1 . The $i^{t h}$ location in the vector corresponding to task $j$ is 1 if and only if task $i$ is a (transitive) predecessor of task $j$ or if $i=j$. This allows for checking causality between any task and a given task $j$ by simply inspecting the vector associated with task $j$-without having to examine predecessor links transitively.

Figure 2 shows basic actions of the verification algorithm. The vector clocks are initialized and updated using a predecessor relationship in lines 6-9. Expressly, once a task $T$ starts execution, we first calculate its vector clock by performing a logical OR operation (reflected with the union operator) between $T$ 's vector clock (initially all $0 \mathrm{~s}$, except for one location) and the vector clocks of its immediate dependence predecessors. Prior to this, we also track information on the dependence successors and recycle successors for each task as shown in lines 2-3 of Figure 2.

In line 12, we obtain $T$ 's recycling predecessor (per the recycling function currently being evaluated). Lines 17-19 ensure that $T$ recycling Trec does not violate our first constraint. This is accomplished by checking if there is a happens-before relation between $T$ and Trec, as well as between $T$ and Trec's immediate dependence successors. As indicated previously, a simple inspection of $T$ 's vector clock can determine this (in the implementation of the assert function in lines 17 and 19).

Recall that we do not assume prior information on a task's successors. Instead, we track each use as shown in line 31. This implies that Trec's dependence successors list may not contain all of its successors when $T$ makes the causality check in lines 1819 (certain successors could consume Trec's output later in the execution after Trec's output is overwritten by $T$ ). To detect such cases, a task's dependence successors retroactively check (performed at the access time) if the data item has been already overwritten or not. This is reflected in line 33. The idea is to ensure that a dependence predecessor's recycling successors list is empty.

All of the preceding logic is for checking the first constraint. Violation of the second constraint by a recycling operation is detected in lines 21-27. Specifically, if the intended recycle predecessor Trec has been already recycled by another task, we perform the same checks between $T$ and the previous recycle successors maintained in Trec.recycle_succ.

One important aspect to note is that the algorithm itself executes concurrently on different threads processing the tasks. To avoid race conditions while updating the data structures associated with the algorithm, steps in lines 14, 24, and 31 are performed atomically. Also, the algorithm is independent of the specifics of the task scheduler employed and, therefore, is compatible with any task graph scheduler. Atomic statements only operate on individual fields or task descriptors and are implemented in a lock-free fashion using atomic instructions (where possible). 


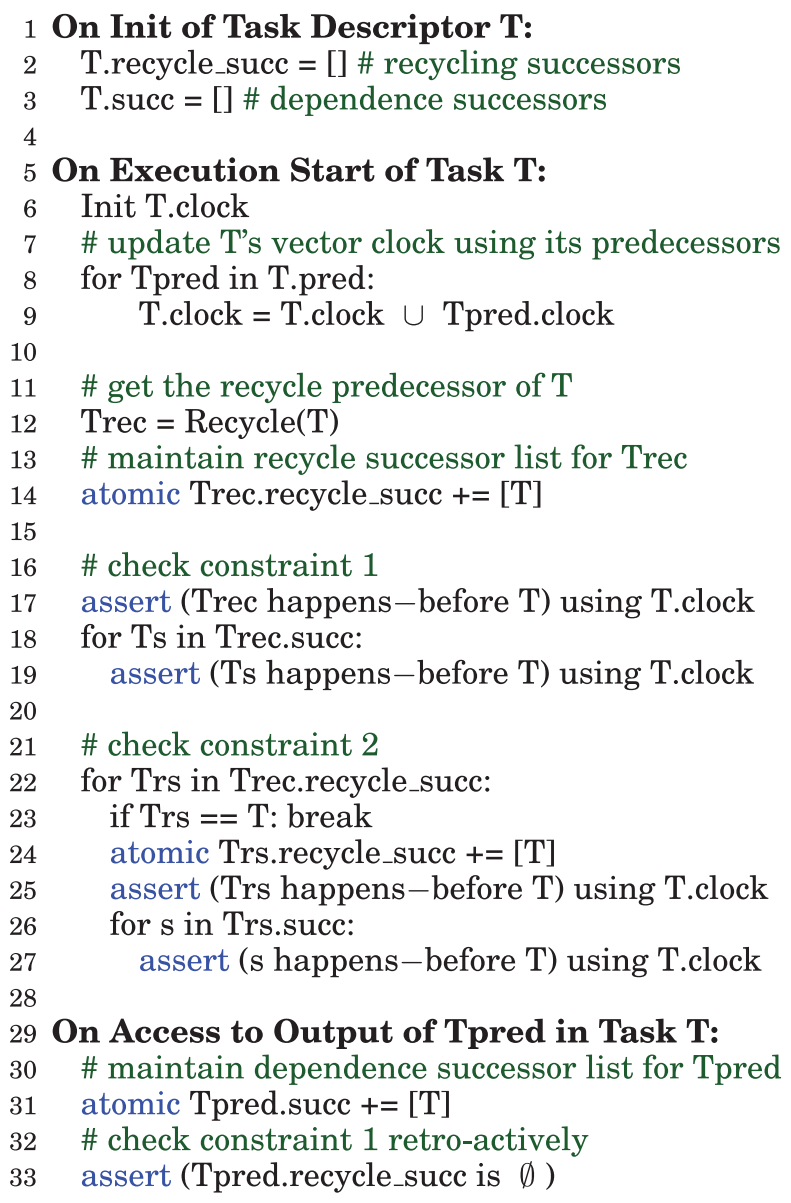

Fig. 2. Store Recycling Verification Algorithm.

Note that the verification algorithm's actions are specified in terms of individual tasks, independent of the execution order. Therefore, the algorithm can be incorporated into any task graph scheduling strategy. In this article, we employ a demand-driven task graph scheduling strategy (explained in Section 6). The dynamic scheduling strategy employed can result in different runtime behavior due to variations in task execution times, system noise, and randomness in the scheduler. However, as discussed in more detail in Section 7, we did observe consistent runtime performance with low noise.

Evaluating Multiple Recycling Functions. The algorithm to verify a recycling function does not require that the same recycling function be employed by the task scheduler. We exploit this property to verify multiple recycling functions simultaneously. In particular, the recycle_succ field in Figure 2 is replaced with a vector with one field per recycling function evaluated. All candidate recycling functions are evaluated simultaneously by independently updating the recycle successors for each recycling function.

In the absence of a recycling function, the total memory usage is the entire size of the data items produced by all tasks in the task graph. A store recycle operation to a valid task avoids the allocation of new memory. Therefore, total memory usage when executing a task graph under a store recycling function is inversely proportional to 


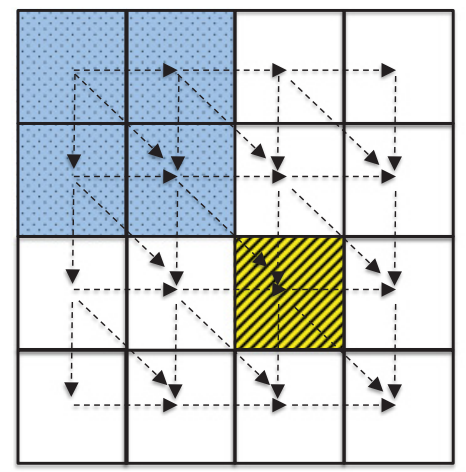

(a)

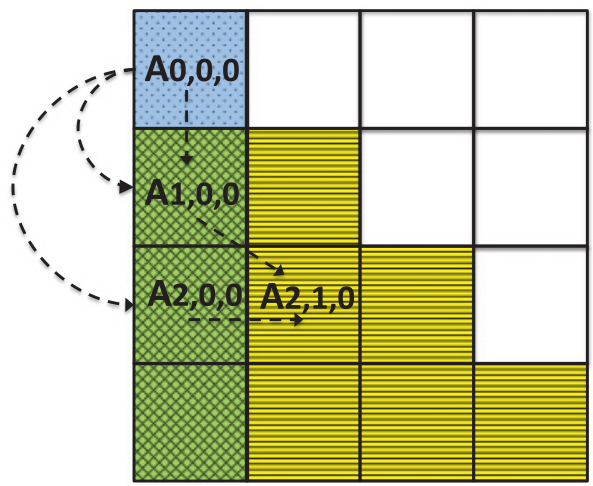

(b)

Fig. 3. (a) Task graph for LCS. Blue tasks (filled with dots) form the set of candidates whose output the yellow task (filled with diagonal lines) can recycle. (b) Examples for three different task classes in Cholesky shaded in different colors.

the number of recycling operations performed, and the amount of memory allocation avoided. Therefore, we track the number of recycle operations for each recycle function being evaluated. If multiple recycle functions happen to be correct, we choose the recycling function with minimal memory usage for subsequent executions of the task graph.

\subsection{Exploring the Space of Recycling Functions}

Our next goal is to automatically derive recycling functions. Our approach here involves first generating a set of candidate recycling functions, then verifying that they lead to correct execution. Our first observation is that candidates a given task can recycle safely should be a subset of the task's immediate and transitive predecessors. Figure 3(a) demonstrates a small task graph example for Longest Common Subsequence (LCS). In the figure, an edge from task A to task B denotes the dependence of task B on task A. LCS is a simple application and uncomplicated (loop-based) programming models are also sufficient to support efficient execution. While it is not one of the benchmarks we use in our experimental evaluation, it is a useful first example to demonstrate reuse functions. Specifically, in this application, each task consumes data items that are produced by its left, upper, and diagonal neighbors. In the figure, the blue tasks' outputs (filled with dots) can be recycled by the yellow task (filled with diagonal lines). In general, such candidates for a task can be explored by traversing the task graph, starting from the given task and exploring the task graph toward the source tasks (tasks with no incoming dependences) by using predecessor relations provided by the user.

Our procedure works as follows. First, we require information about the task graph's dependence structure from the user. The dependence structure of a task graph consists of the number of immediate predecessors for any task and a function to reach each of them. Next, the paths are enumerated to a bounded depth, referred to as hops, to limit the cost of verification.

We next explain the process using LCS, and a more complex application, Cholesky. Table I summarizes the necessary information that a user provides as the dependence structure of tasks in LCS. Each task in LCS generally depends on three tasks referred to as LEFT, UpPER, and DiAg. Therefore, the user provides three functions to reach the 
Table I. Dependence Structures for LCS and Cholesky. Each Tag (such as LEFT, UPPER, ...) in the Dependence Structures Corresponds to Functions Provided by a User to Reach a Specific Predecessor

\begin{tabular}{llc}
\hline Benchmark & Task Classes & $\begin{array}{c}\text { Dependence } \\
\text { Structure }\end{array}$ \\
\hline \multirow{2}{*}{ LCS } & lcs & lcs: (LEFT, \\
& UPPER, \\
& DIAG) \\
\hline \multirow{4}{*}{ Cholesky } & cholesky & cholesky:(MULT) \\
& trisolve & trisolve:(MuLT, \\
& mmult & CH) \\
& & mult:(MULT, \\
& & TRI1 \\
& & TRI2) \\
\hline
\end{tabular}

Table II. Examples of Automatically Explored Recycling Functions with a 2-hop Predecessor Traversal for LCS and Cholesky

\begin{tabular}{|c|c|c|}
\hline Recycling Functions & LCS & Cholesky \\
\hline func0 & $\begin{array}{c}\text { lcs: (hop1 to DIAG, } \\
\text { hop2 to LEFT) }\end{array}$ & $\begin{array}{l}\text { cholesky: (hop1 to MuLT) } \\
\text { trisolve: (hop1 to MULT) } \\
\text { mmult: (hop1 to MULT) }\end{array}$ \\
\hline func1 & $\begin{array}{l}\text { lcs: (hop1 to DIAG, } \\
\text { hop2 to UPPER) }\end{array}$ & $\begin{array}{l}\text { cholesky: (hop1 to MuLT) } \\
\text { trisolve: (hop1 to MuLT) } \\
\text { mmult: (hop1 to MULT, } \\
\text { hop2 to MULT) }\end{array}$ \\
\hline func2 & $\begin{array}{c}\text { lcs: (hop1 to DIAG, } \\
\text { hop2 to DIAG) }\end{array}$ & $\begin{array}{c}\text { cholesky: (hop1 to MULT) } \\
\text { trisolve: (hop1 to MULT, } \\
\text { hop2 to MuLT) } \\
\text { mmult: (hop1 to MULT) }\end{array}$ \\
\hline
\end{tabular}

LEFT, UPPER, and DiAg predecessor for any given task. We look for predecessors of a task reachable from that task using any of the three functions within a bounded number of hops. Table II depicts some example recycling functions for LCS that are explored automatically (with a 2-hop search) using this information.

The next example is Cholesky, a more complex application because, unlike LCS, all tasks in execution do not perform similar operations (Cholesky is one of the applications used in our evaluation). For Cholesky, we observe that tasks in a task graph often can be organized into groups with all tasks in a group sharing the same data access patterns, number of predecessors, and ordering relationships carrying out similar calculations. We refer to each group as a task class. A typical parallel implementation of Cholesky factorization consists of three different task classes as illustrated in Figure 3(b): (1) Cholesky algorithm on diagonal blocks (cholesky), (2) triangular solve operation on blocks under diagonal (trisolve), and (3) matrix-matrix multiplication for other blocks below the diagonal (mmult). In the figure, these tasks are represented with blue (filled with dots), green (filled with outlined diamonds), and yellow (filled with horizontal lines) colors, respectively, and tasks $A_{0,0,0}, A_{1,0,0}, A_{2,0,0}$, and $A_{2,1,0}$ are labeled as representatives for each task class. Clearly, $A_{2,0,0}$ and $A_{2,1,0}$ have different dependence structures. In such cases, a single dependence structure provided by a user would not be sufficient to represent the structure of different task classes. If only a single dependence structure is provided, the traversal path represented by the chosen recycling function would not be applicable to some tasks. This would cause these tasks to allocate new memory for their output and increase memory consumption significantly because of missed recycling opportunities. This issue can be handled by providing a dependence structure for each task class in an application. If there is more than one task class in the task graph, we explore the possible set of recycling functions for each task class, then generate combined recycling functions. Table I shows an example dependence 
structure provided for LCS and Cholesky, while Table II illustrates the combined recycling functions generated automatically.

\section{PRODUCTION RUNS WITH INCORRECT RECYCLING FUNCTIONS}

The algorithm in Section 4 identifies candidate recycling functions for arbitrary execution schedules of a given task graph. Programs often produce structurally similar but not identical task graphs for different inputs or problem sizes. Thus, a recycling function valid for one problem size (or input) might not be valid for a different input or problem size. Repeated verification of a candidate recycling function for every input to a program can be prohibitively expensive, especially for large problem sizes and in production runs. In this section, we present our approach to ensure continued correct and efficient execution of a task graph-despite the possibility of the chosen recycling function being invalid.

Although we can detect incorrect recycling functions using vector clocks, we remove vector clocks and related mechanisms during normal execution to minimize performance impact. Instead, the following two guarantees are maintained using other mechanisms:

\section{GUARANTEE 1. A recycling function does not result in concurrent recycling.}

GUARANTEE 2. A data item recycled before all of its uses are done is correctly computed again by re-execution of its producer task.

The first requirement (Guarantee 1 ) implies that a recycling function might map two different tasks, e.g., $B$ and $C$, onto the same task $A$. If task $B(C)$ does not causally precede task $C(B)$, this mapping results in a data race. To detect concurrent recycling efficiently, we hold an additional active_write flag in each allocated memory (for a task's output) and protect it with a lock for synchronized access. When a task is about to perform a valid recycle operation, it raises this flag as an indication that memory for a corresponding data item is currently being written by a task for its output. During the time the active_write flag is raised, any other task trying to recycle the same data item checks the flag's value, notices that it is currently in use, and allocates new memory. Once the task that raises the flag (i.e., the current definition of the data item) finishes execution successfully, it changes the flag's value back to false for future recycle operations on the same data item.

Figure 4 illustrates how concurrent recycling on the same data item is detected. In both Figures 4(a) and 4(b), completed tasks $(A, E$, and $F$ ) are shown in green and filled with diamonds, whereas yellow and dot-filled circles represent tasks $(B)$ that are currently executing. In Figure 4(a), fields shown for $A$ indicate that $A$ 's output resides in data item $D$, and $A$ 's output has not yet been recycled. $B$ wants to recycle $A$ 's output and does so successfully. The change in system state after the recycle operation is shown in Figure 4(b). After the recycle operation is completed, another task $C$ attempts to recycle $A$ 's output, but fails to do so because $D$ 's active_write flag ("awrite" in the figure) indicates that another task $(B)$ is currently overwriting $D$. As a result, $C$ allocates new memory for its own output.

Now, we focus on the second guarantee. A data item might be recycled by a task even if there are remaining successors that will access the data item later in the execution. We guarantee correct execution in these scenarios by employing an overwrite detection and an accompanied re-execution mechanism at runtime. Each task holds an additional recycled flag that indicates if the task's output has been overwritten by another task. To detect an early overwrite, each task checks the recycled flag of all of its immediate predecessors before and after it performs its main computation. If the flag is set to true for any of them, the task resets itself and starts a re-execution procedure for its 


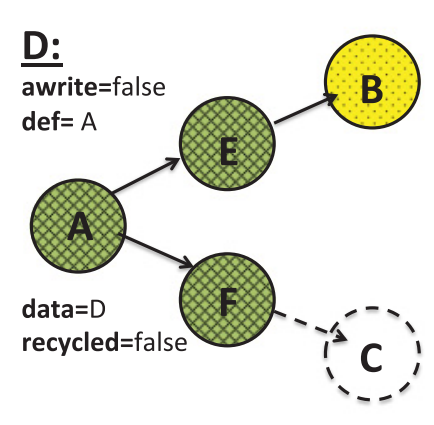

(a) before recycling

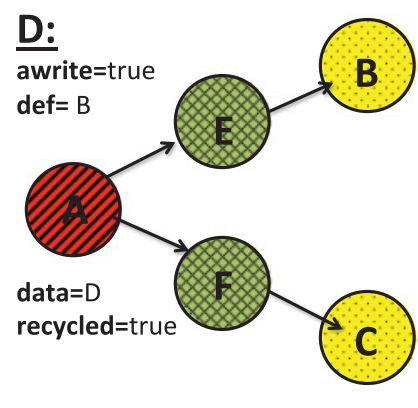

(b) after recycling

Fig. 4. Concurrent recycle detection on an example task graph. (a) and (b) show the system state before and after task $B$ recycles task $A$ 's data item $D$. Task $C$, trying to recycle the same data item, is forced by the runtime to allocate new memory.

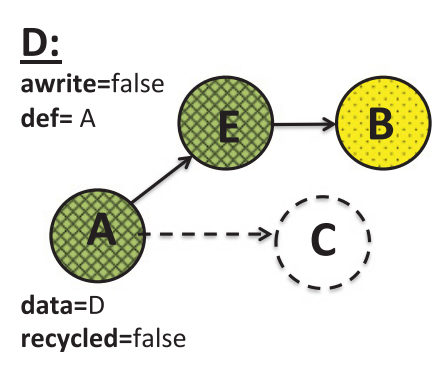

(a) before recycling

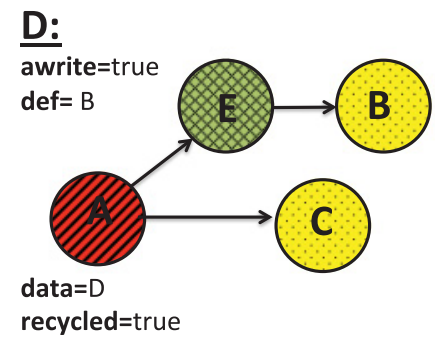

(b) after recycling

Fig. 5. Eager recycle detection on an example task graph. (a) and (b) show system state before and after task $B$ recycles task $A$ 's data item $D$. $C$ detects that $A$ 's output has been recycled and initiates its re-execution.

immediate predecessors whose outputs have been overwritten. Note that, during this time, no other task can recycle the data item that the task itself is recycling because active_write of the corresponding data item would not be changed back to false until the task successfully finishes its own execution.

Figure 5 illustrates an example scenario for detection of a data item that has been recycled too soon. Different colors and fill patterns correspond to the same task status as depicted in Figure 4, where Figure 4(a) shows the runtime state before $B$ recycles $A$ 's output stored in $D$. After the recycling operation, with the corresponding state shown in Figure 4(b), $C$ tries to access the data produced by its immediate predecessor $A$. Because $A$ 's output already has been recycled, $C$ detects the recycle operation by checking A's recycled flag and initiates re-execution of $A$.

In addition to providing correct execution, regardless of the recycling function being used, the execution should proceed in an efficient manner. If a recycling function is observed to lead to concurrent recycling or task re-executions, we switch to the recycling function that results in the next largest number of recycles. That way, we do not 
continue to pay re-execution costs by employing the same incorrect recycling function for the rest of the execution.

\section{IMPLEMENTATION}

In this section, we demonstrate an implementation of the algorithms presented in Sections 4 and 5 in the context of an existing task graph scheduling framework: NABBIT [Agrawal et al. 2010]. NABBIT is a provably time-efficient task graph scheduler using work stealing. In this section, we briefly describe task graph scheduling performed by the NABBIT framework and explain the extensions to support our recycling algorithms.

\subsection{Task Graph Scheduling using NABBIT}

In NABBIT, each task in a computation is referred by a task key. This task key is a unique identifier to relate different references to the same task without the need for a pre-allocated task object. The user provides two functions to the runtime: one to return a list of immediate predecessors of a task (given its key) and the other to define the computation to be performed by each task. The runtime stores a task descriptor for each task created during the execution. A task descriptor primarily comprises a join counter to track the number of outstanding predecessors for a task; a notifyArray to store successors that are to be notified once the task finishes its execution; status to represent execution status of the task at the moment; and a data pointer that points to the memory region, which the task uses to produce its output.

Task graph execution is orchestrated by the runtime through a concurrent hash map. A task descriptor is inserted into the hash map for each task created during execution. Execution of a task graph begins with the insertion of a sink task (task with no outgoing dependences) into the hash map. Each inserted task first traverses its predecessors and checks their status. For each predecessor whose task descriptor does not exist in the hash map, a new task descriptor is created. This way, the task graph is expanded in a recursive fashion until a source task (task with no incoming dependences) is found. If a predecessor is to be computed by another thread, the task registers itself in the predecessor's notifyArray, so it can be notified once the predecessor finishes execution. Each time a predecessor completes, join of the task is decremented. Tasks are scheduled for execution once their join counter reaches zero. Execution of a task graph terminates once the sink task completes its execution.

The execution of the task graph from the sink vertex is referred to as demand-driven execution. Note that the memory to store the data block produced by a task is not allocated until the task is ready to execute (when all of its predecessors have been executed). Therefore, the memory requirement is not necessarily related to the task graph's critical path length. In some scenarios, demand-driven execution might be more memory efficient compared to data-driven execution [Kambadur et al. 2009].

\subsection{Extensions to the NABBIT Scheduler}

The evaluation of recycling functions is implemented in the NABBIT scheduler by augmenting the relevant events shown in Figure 2. To detect incorrect recycle operations, we hold an additional boolean field in each task descriptor, referred to as recycled. Once a task's output data item has been recycled, its recycled flag is raised. This task's successors would observe the incorrect recycle operation by checking the value of the flag either when they first traverse the task, before they perform their respective computation, or after they finish their computation.

Upon observation, a successor initiates re-execution of the overwritten predecessor. First, the predecessor's current task descriptor is replaced with a new one, where each field is assigned to its initial value. The predecessor to be re-executed traverses its own dependences and transitively re-executes any predecessors whose output has also been 
recycled. Like any other task, a re-executed task performs its computation when its join counter reaches zero. Upon successful completion, it notifies the successors that initially observed the incorrect recycle operation.

To provide a low overhead re-execution mechanism, we must ensure that the producer task of a recycled-but still needed-data item is re-executed only once (and not by every observer of the incorrect recycle operation). We associate each task descriptor with a life number. When multiple consumers of a data item detect incorrect recycling, each consumer checks if a re-execution procedure has already been initiated for the producer task with the current life number. The first consumer that observes this condition is the only one to perform the actual re-execution. To facilitate this, we maintain a separate hash table that associates a given task with its most recent life number that has been re-executed. Note that this hash table is initially empty, and a record for a given key is inserted only if a task needs to be re-executed.

\section{EXPERIMENTAL EVALUATION}

\subsection{Experimental Setup}

We evaluate our approach on Intel Xeon Phi 7110P (Knights Corner) many-core coprocessor that has 61 cores running at $1.1 \mathrm{GHz}$ with a $32 \mathrm{~KB}$ L1 cache, $512 \mathrm{~KB}$ L2 cache per core, and 8GB device memory for all cores. Xeon Phi represents a system with a large number of cores and relatively modest memory per-core, two characteristics expected to be very common among nodes of future supercomputers. Thus, the Xeon Phi was an ideal choice for our experiments. All benchmarks were compiled using the Intel icc-13.3.163 compiler with -O3 optimization. All experiments were executed in the $n a$ tive mode directly on a many-core coprocessor without sharing any work with the host processor. We evaluated the benchmarks on up to 244 threads. For thread counts at or lower than 61, we employed one thread per processor core. For the 244-thread experiments, we employed four threads per code, exploiting the hardware threads available on the Xeon Phi. Note that application characteristics will strongly influence whether or not using more threads than cores improves performance.

The the scheduler's dynamic nature can result in distinct runtime behavior for different runs. All experimental data we report are the average of five separate executions. We also show standard deviation as the error bar for the small fraction of cases where the standard deviation is non-trivial. In the remainder of this section, we compare executions with (a) no data reuse (referred to as single-assignment), (b) memory reclamation based on information on the number of uses for each task's output (referred to as use-count), (c) hand-optimized implementations in NABBIT (referred to as handwrittenrecycle), and (d) our approach to automated recycling function selection (referred to as auto-recycle). The hand-optimized versions were produced by studying each benchmark and deriving solutions specific to each one.

\subsection{Benchmarks and Applicability of Use-Count-Based Garbage Collection}

We used seven benchmarks for our evaluation studies: Smith-Waterman (SW), the local sequence alignment algorithm [Low et al. 2007]; Floyd-Warshall (FW), all-pairs shortest path algorithm in a weighted graph; two dense linear algebra kernels, LU decomposition and Cholesky factorization; Rician Denoising [Bui et al. 2012], an image processing benchmark; and two Rodinia benchmarks, Srad and Hotspot [Che et al. 2009]. Table III shows the configuration for all benchmarks.

The use-count-based garbage collection scheme, a standard technique for memoryoptimized execution of task graphs, is a candidate approach for comparison against our method. This technique is supported in existing runtimes, such as Intel CnC [Budimlić et al. 2010], and requires a tuning interface for the programmers to specify the number 
Table III. Matrix size (N), Block Size (B), Total Number of Tasks (T), and Number of Task Classes (C) for each Benchmark. "(d1)" and "(d2)" Represent Different Problem Sizes

\begin{tabular}{lrrrr}
\hline & \multicolumn{1}{c}{$\mathbf{N}$} & \multicolumn{1}{c}{ B } & \multicolumn{1}{c}{ T } & C \\
\hline Cholesky (d1) & $5 \mathrm{~K} \times 5 \mathrm{~K}$ & $128 \times 128$ & 11,480 & 3 \\
Cholesky (d2) & $10 \mathrm{~K} \times 10 \mathrm{~K}$ & $128 \times 128$ & 88,560 & 3 \\
FW (d1) & $2.5 \mathrm{~K} \times 2.5 \mathrm{~K}$ & $128 \times 128$ & 8,000 & 4 \\
FW (d2) & $5 \mathrm{~K} 5 \mathrm{~K}$ & $128 \times 128$ & 64,000 & 4 \\
Hotspot (d1) & $8 \mathrm{Kx} 8 \mathrm{~K}$ & $128 \times 512$ & 10,240 & 1 \\
Hotspot (d2) & $16 \mathrm{~K} \times 16 \mathrm{~K}$ & $128 \times 512$ & 40,960 & 1 \\
LU (d1) & $5 \mathrm{~K} \times 5 \mathrm{~K}$ & $128 \times 128$ & 22,140 & 4 \\
LU (d2) & $10 \mathrm{~K} \times 10 \mathrm{~K}$ & $128 \times 128$ & 173,880 & 4 \\
Rician (d1) & $256 \times 256 \times 256$ & $32 \times 32 \times 32$ & 10,240 & 2 \\
Rician (d2) & $512 \times 512 \times 512$ & $32 \times 32 \times 32$ & 81,920 & 2 \\
Srad (d1) & $2 \mathrm{~K} \times 2 \mathrm{~K}$ & $128 \times 256$ & 2,570 & 3 \\
Srad (d2) & $8 \mathrm{~K} \times 8 \mathrm{~K}$ & $128 \times 256$ & 40,970 & 3 \\
SW (d1) & $3 \mathrm{Kx} 3 \mathrm{~K}$ & $128 \times 128$ & 17,576 & 2 \\
SW (d2) & $6.2 \mathrm{~K} \times 6.2 \mathrm{~K}$ & $128 \times 128$ & 132,651 & 2 \\
\hline
\end{tabular}

of uses associated with each data item. The approach works by simply decrementing the use count of a data item after each access on it-once the use count reaches zero, memory allocated for the data item is reclaimed.

Compared to this approach, the main advantage of our technique applies to programmer productivity and its broader applicability, especially for applications where use count is not statically available. Thus, before reporting performance comparisons, we discuss the applicability and programming effort required on our target set of benchmarks. It turns out that applying use-count-based memory reclamation to the benchmarks being evaluated demands significant additional effort from the programmer. For linear algebra kernels LU and Cholesky, specification of linear functions to determine the exact use count of each data item is required. Moreover, special attention must be directed to any boundary conditions to avoid over-specification of use counts, which can lead to memory not being deallocated until the task graph completes execution. An existing study [Budimlic et al. 2008] to automate extraction of use counts in Intel $\mathrm{CnC}$ also observes that determining correct use counts can be a significant challenge for programmers.

In some cases, the exact number of data item uses might be unknown, even at runtime. For example, after each iteration, Rician denoising performs a global convergence check via a reduction on convergence status of all data items. The convergence check is accelerated with a short-circuiting evaluation, which initiates the next iteration immediately if any data items have changed significantly. The introduction of shortcircuiting for performance eliminates the need to access the convergence status of all data items. However, it also implies that the number of accesses on these elements cannot be determined a priori. Thus, overall, our recycling approach eliminates the need for use counts and, in the process, broadens the set of applications that can be efficiently supported while also minimizing the programmer's required involvement.

\subsection{Comparison Between Single-Assignment and Recycling}

Our first experiment compares each benchmark's performance with single-assignment (new memory allocation for each task execution) and memory-recycling-based implementations. The goal of this experiment is to demonstrate the memory efficiency of recycling-based implementations. For this comparison, we refer to Figure 6((a) through (n)). The single-assignment version allocates a new data item for each task in execution, leading to a high memory footprint for each benchmark. In one of the memory recycling 


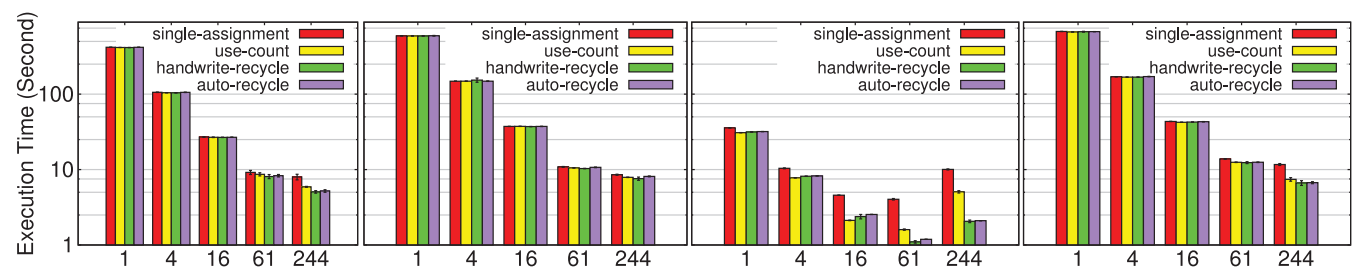

(a) Cholesky(d1)

(b) FW (d1)

(c) Hotspot (d1)

(d) LU (d1)

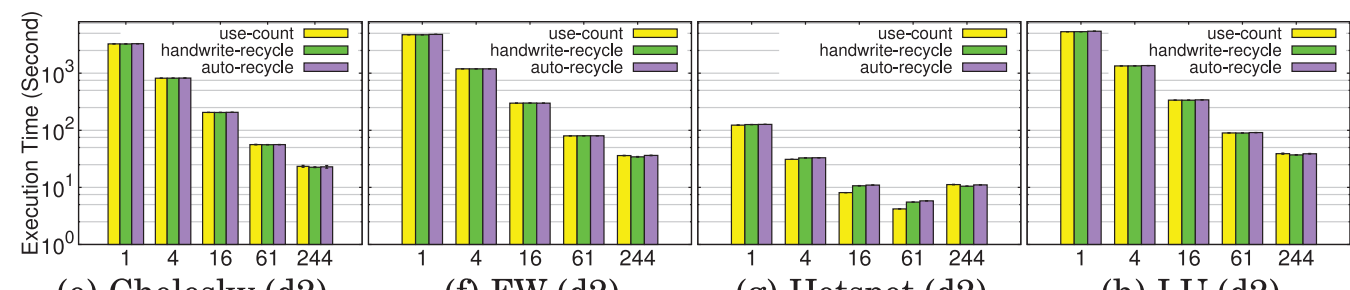

(e) Cholesky (d2)

(f) FW (d2)

(g) Hotspot (d2)

(h) LU (d2)

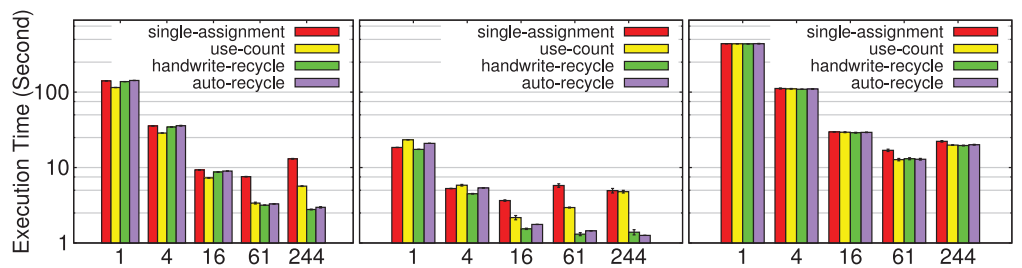

(i) Rician (d1)

(j) Srad (d1)

(k) SW (d1)

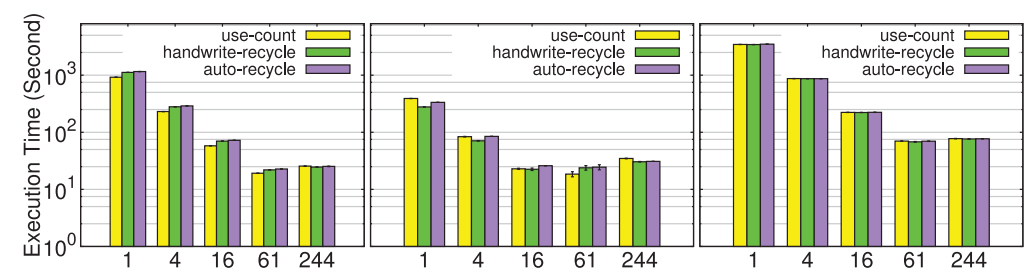

(l) Rician (d2)

(m) Srad (d2)

(n) SW (d2)

Fig. 6. Execution time of each benchmark with single-assignment, use-count, optimized handwrittenrecycle, and auto-recycle versions. Performance of all benchmarks are evaluated on two different problem sizes, labeled as "(d1)" and "(d2)." Execution of single-assignment versions in Cholesky (d2), FW (d2), Hotspot (d2), LU (d2), Rician (d2), Srad (d2), and SW (d2) did not finish as they ran out of memory. Figures (a)-(d), (e)-(h), (i)-(k), and (l)-(n) share the same y-axis, respectively.

versions (labeled as "handwrite-recycle"), the user directly implements recycling operations in the application code. We created this version as it represents the best performing baseline against which our automated recycling schemes are evaluated later in this section. We also evaluated use-count-based garbage collection and reported the results with bars labeled as "use-count." Finally, Figure 6 also includes a comparison with "auto-recycle" versions, but details are covered in the next subsection. We run each benchmark with a small and a large problem size. These instances are distinguished by "(d1)" and "(d2)" labels following the benchmark name in each subfigure.

On large problem instances, the single-assignment version cannot even finish execution because of out-of-memory errors, so no bar is shown for this version in the corresponding figures. Focusing first on the "handwrite-recycle" versions, this version completes execution successfully in all cases, and continues to scale well for almost all benchmarks. Specifically, it achieves (over 1 thread versions) 144.3x speedup for 
Table IV. Memory Footprint (in GB) of Single-assignment ("Single"), Handwritten-recycle ("Hand"), and Auto-recycle Versions that Lead to Minimum ("Min-Auto") and Maximum ("Max-Auto") Memory Footprint

\begin{tabular}{lrccc}
\hline & Single & Hand & Min-Auto & Max-Auto \\
\hline Cholesky (d2) & 11.60 & 0.42 & 0.42 & 0.84 \\
FW (d2) & 8.38 & 0.21 & 0.61 & 1.19 \\
Hotspot (d2) & 10.73 & 2.14 & 2.14 & 2.41 \\
LU (d2) & 22.79 & 0.83 & 0.83 & 1.65 \\
Rician (d2) & 21.47 & 3.22 & 3.22 & 4.36 \\
Srad (d2) & 30.7 & 4.82 & 4.82 & 5.42 \\
SW (d2) & 8.69 & 0.17 & 0.17 & 0.34 \\
\hline
\end{tabular}

Cholesky (244 threads), 137.3x for FW (244 threads), 22.7x for Hotspot (61 threads), 144.1x for LU (244 threads), 49.6x for Rician ( 244 threads), 13.2x for Srad (61 threads), and $51 \mathrm{x}$ for SW (61 threads). On smaller problem instances, the single-assignment version completes, but its performance degrades with increasing numbers of threads. This trend can be clearly seen in Cholesky (d1), Hotspot (d1), LU (d1), Rician (d1), and Srad (d1) instances. After a cache behavior analysis, we observed that L1 cache misses and the number of data references for the single-assignment version increase considerably with increasing numbers of threads, leading to performance slowdowns. In terms of better performance and the ability to handle larger problem sizes, memory recycling clearly is a better alternative to the single-assignment scheme.

In comparing "use-count" and "handwrite-cycle," results show that the former performs no better than the latter. As previously emphasized, the key advantage of our method is its wider applicability and programmer productivity. However, in achieving these benefits, there is no performance loss over the use-count-based garbage collection scheme.

\subsection{Auto-Recycling Overheads}

Our preceding discussion has demonstrated the advantage of memory recycling over single assignment. The next question is the effectiveness of automated recycling versions. To this end, we measure the performance of our scheme, which is shown by the "auto-recycle" bars in Figure 6, and calculate its overheads over handwritten-recycle versions. The auto-recycle versions for these experiments were generated by first exploring recycling functions automatically with a 2-hops traversal, then employing the one that leads to the most simulated recycling operations. At 61 threads, our scheme leads to $0.3 \%, 0.2 \%, 4.9 \%, 1.8 \%, 3 \%, 2.6 \%$, and $2.6 \%$ overheads over the handwrittenrecycle version for Cholesky (d2), FW (d2), Hotspot (d2), LU (d2), Rician (d2), SW (d2), and Srad (d2), respectively. These small overheads demonstrate that the recycling approach can be effectively automated. These overheads stem from synchronization required to detect concurrent and premature recycling in the automatic versions, as well as a more direct mapping and avoidance of indirect references in the handwritten versions.

To further demonstrate the effectiveness of our methods for automatically generating recycling functions, Table IV reports memory footprint (in GB) of single-assignment, handwritten-recycle, and auto-recycle versions. Memory footprint is calculated based on the number of allocated data blocks for intermediate and final results. Because we have several different auto-recycle versions (which can have different memory requirements), the table reports memory footprint of the function(s) that lead to the minimum ("Min-Auto") and maximum ("Max-Auto") memory. In most benchmarks, except FW, we are able to generate recycling functions that consume the same amount of memory as the handwritten-recycle versions. Recall that from the set of recycling 


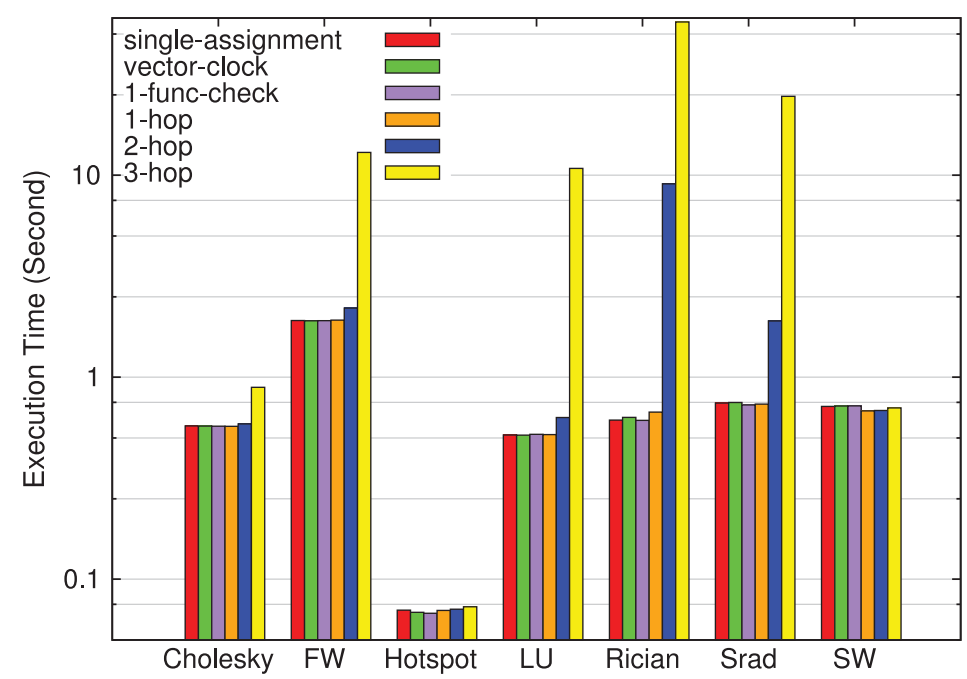

Fig. 7. Costs associated with verification of recycling functions at 61 threads.

functions found to be correct, we choose the version with the most reuse. We observe that in all benchmarks, minimum consumption is achieved by the version we selected using the aforementioned logic. We also compared the reuse count in the verification step and the actual memory footprint of each generated recycling function, and found an inverse correlation. Overall, the data presented in Figure 6 and Table IV distinctly demonstrate that automated generation of the recycling function is effective in terms of memory requirements and execution time.

In FW, the hand-optimized implementation reduces memory usage via additional ordering constraints that are not implied by the dependence predecessors. In particular, the implementation begins with a two-dimensional matrix and explicitly orders tasks that perform subsequent updates to the same matrix block. Our approach is only based on data dependences. Thus, it cannot match the manually optimized FW's memory overhead. Note that memory usage is still considerably lower than the singleassignment version. The auto-recycle version can match the hand-optimized version if the user provides additional dependences when candidate recycling functions are explored.

\subsection{Recycling Function Verification Costs}

In Figure 7, we evaluate the costs associated with verifying a set of candidate recycling functions. For simplicity, data are only reported for the 61-thread execution. The reported costs primarily comprise vector clock calculation and verification of recycling functions with the help of vector clocks. Problem sizes used during verification, given in Table V, are much smaller than those in actual runs because the goal is to determine the appropriate recycling functions with low overheads before large-scale runs.

In Figure 7, the first bar ("single-assignment") represents a baseline execution without any recycling, vector clock calculations, or function verification. The remaining bars respectively show the time it takes to calculate vector clocks on top of the singleassignment version, as well as the time it takes to verify a single correct recycling function provided by the user ("1_func_check") and the recycling functions explored automatically by 1 ("1_hop"), 2 ("2_hop"), and 3 ("3_hop") hops. Note that the last four bars for each benchmark also include the cost for vector clock calculation, which is an integral part of the verification scheme. Table VI reports how many recycling functions 
Table V. Matrix Size (N) and Block Size

(B) used for each Benchmark for

Recycling Function Verification

\begin{tabular}{lrr}
\hline & \multicolumn{1}{c}{$\mathbf{N}$} & \multicolumn{1}{c}{$\mathbf{B}$} \\
\hline Cholesky & $1 \mathrm{~K} \times 1 \mathrm{~K}$ & $128 \times 128$ \\
FW & $1 \mathrm{~K} \times 1 \mathrm{~K}$ & $128 \times 128$ \\
Hotspot & $1 \mathrm{~K} \times 1 \mathrm{~K}$ & $128 \times 512$ \\
LU & $1 \mathrm{~K} \times 1 \mathrm{~K}$ & $128 \times 128$ \\
Rician & $64 \times 64 \times 64$ & $16 \times 16 \times 16$ \\
Srad & $1 \mathrm{~K} \times 1 \mathrm{~K}$ & $128 \times 256$ \\
SW & $512 \times 512$ & $128 \times 128$ \\
\hline
\end{tabular}

Table VI. Number of Recycling Functions Checked and Actually Verified as Correct When Recycling Functions are Explored Automatically with 1, 2, and 3 Hops Each pair " $n_{1} / n_{2}$ " denotes that $n_{1}$ functions are deteermined to be correct after considering $n_{2}$ functions.

\begin{tabular}{lrrr}
\hline & 1-hop & \multicolumn{1}{c}{ 2-hops } & \multicolumn{1}{c}{ 3-hops } \\
\hline Cholesky & $1 / 6$ & $12 / 240$ & $114 / 4,400$ \\
FW & $1 / 12$ & $16 / 1,440$ & $192 / 70,400$ \\
Hotspot & $0 / 5$ & $5 / 30$ & $88 / 155$ \\
LU & $1 / 12$ & $16 / 1,440$ & $192 / 70,400$ \\
Rician & $0 / 98$ & $266 / 16,905$ & $3,857 / 100,000$ \\
Srad & $0 / 24$ & $14 / 3,510$ & $2,492 / 100,000$ \\
SW & $1 / 4$ & $6 / 36$ & $18 / 196$ \\
\hline
\end{tabular}

are checked for correct execution by "1_hop," "2_hop," and "3_hop" versions. Results show that vector clock calculation does not incur any visible costs over the baseline. One correct user-provided recycling function is checked for correct execution efficiently without any overheads, and the time it takes to execute the application while verifying functions generated with 1 and 2 hops is quite close to baseline. For the 3-hops case, there is an observable increase in the execution time, especially in FW, LU, Rician, and Srad, where more than 70,000 distinct recycling functions are checked for correct execution. Though it is a high relative cost for execution with a small problem size, in absolute terms, it is still small (about 40 seconds for Rician). The entire verification phase takes less than 1 second for most other benchmarks. Note that the generation of recycling functions is performed sequentially before verification starts, but the time ranges from a few microseconds to 0.035 seconds.

Our analysis of verified recycling functions revealed that in addition to efficiency, our technique is able to filter out incorrect recycling functions with perfect recall and coverage, i.e., there are no false negatives or false positives in the entire process.

\subsection{Re-Execution Overheads}

The cost of determining an appropriate recycling function primarily depends on the re-execution overhead. In the worst case, a recycling function can lead to re-execution of the entire computation. However, re-execution overheads can be significantly reduced by periodically postponing recycling for data blocks that constitute a consistent snapshot. In this section, we try to evaluate the re-execution overheads for the chosen benchmarks without using these types of optimizations.

For every benchmark considered, we observed that all recycling functions verified for the smaller problem size continue to be valid and efficient for larger problem sizes. Here, we evaluate the performance impact when using one of the recycling functions identified as invalid. 


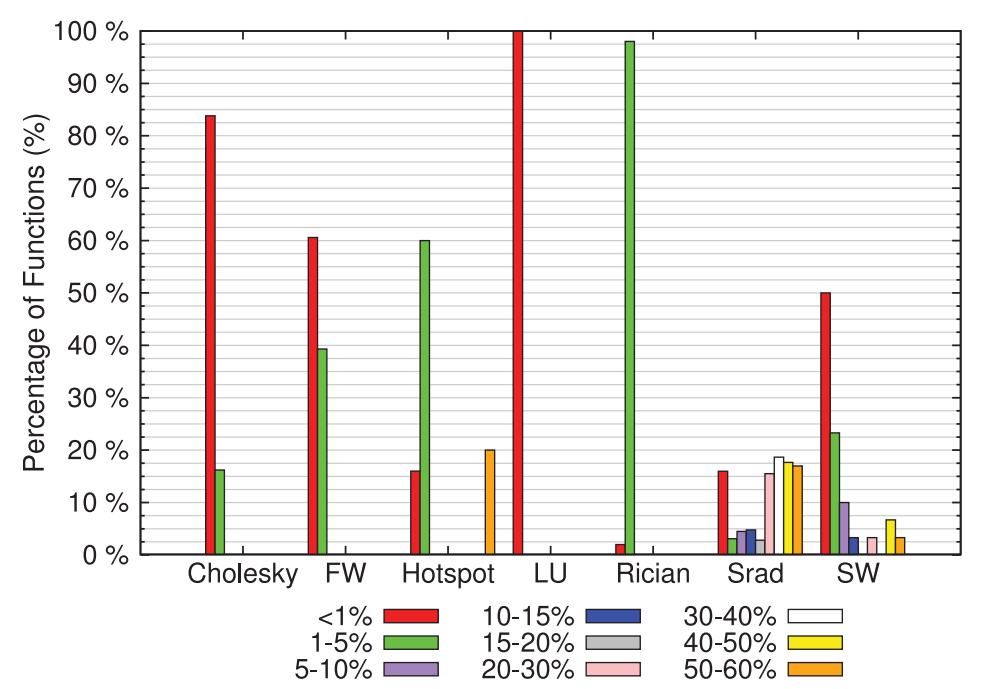

Fig. 8. Distribution of incorrect recycling functions identified for each benchmark in terms of their reexecution overheads (in percentage of original execution time): 228; 1,424;25;1,424;1,500;1,500; and 30 functions are evaluated for Cholesky, FW, Hotspot, LU, Rician, Srad, and SW, respectively.

For each benchmark, we evaluate the impact of all recycling functions that are explored with 2 hops and determined to be incorrect. For Rician denoising and Srad, we picked 1,500 samples from the 16,639 and 3,510 incorrect recycling functions identified within 2 hops. Once an incorrect recycle operation is detected at runtime, we switch to a correct recycling function. We divide possible recovery overheads into nine bins as $<1 \%, 1-5 \%, 5-10 \%, \ldots, 50-60 \%$. Figure 8 shows the number of incorrect recycling functions in each overhead range as a percentage of the total number of incorrect recycling functions evaluated-all executions are with 61 threads. Results show that the number of incorrect recycling functions that cause less than $1 \%$ overhead turns out to be $84 \%, 60 \%, 100 \%$, and $50 \%$ of the total number of incorrect functions for Cholesky, FW, LU, and SW, respectively. The remaining portion of evaluated incorrect functions for most benchmarks results in overheads between 1 and 5\%, whereas it spans almost the entire range of bins for Srad and SW.

Next, we focus on one representative incorrect recycling function and evaluate how the costs vary with different numbers of threads. The chosen incorrect functions map each task in execution to an immediate predecessor whose output has the largest number of usages compared to the other immediate predecessors of the same task. Similar to the previous experiment, once an incorrect recycle operation is detected by runtime, we switch to a correct recycling function for the rest of the execution. Figure 9 shows re-execution overheads over a baseline run, where the chosen recycling function does not lead to any re-executions. For many of the benchmarks, recovery overheads on up to 61 threads are below $1 \%$. We observed that overheads for SW have a large standard deviation, which is shown in the figure as an error bar. In practice, the impact of an incorrect recycling function on execution is non-deterministic. Depending on the schedule resulting from the underlying task graph scheduler in each run, a data item may be recycled too soon before any task finishes its usage on it. In such extreme cases, this forces a significant portion of the task graph to wait on reproduction of the recycled data item, which can significantly hamper the available parallelism in the application. With more threads, this trend becomes visible (as shown by higher recovery overheads at 122 or 244 threads). 


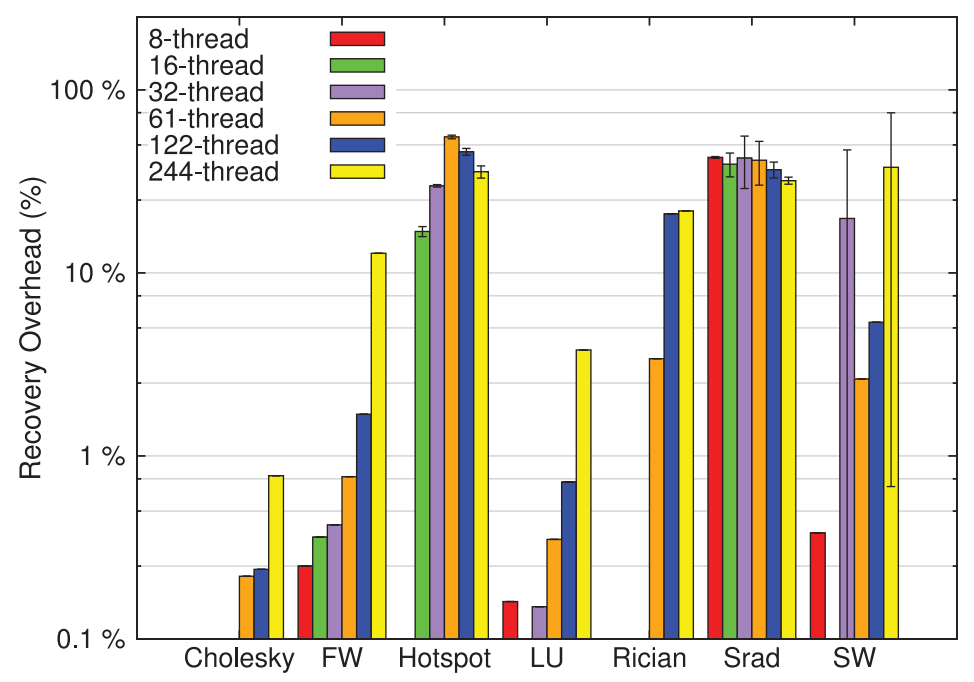

Fig. 9. Re-execution overheads (in percentage) with a representative incorrect recycling function and a varying number of threads.

Hotspot and Srad involve overwrites of a larger fraction of the data items. When attempting to re-execute a task, we often find the task's predecessors also have been overwritten. This transitively leads to re-execution of a large fraction of the total tasks. Therefore, the penalty of an incorrect recycling function is high (up to $55 \%$ in Hotspot and $43 \%$ in Srad) across thread counts.

\section{RELATED WORK}

Data-flow models have existed for four decades [Dennis 1974; Arvind 1981]. A key concept in data flow is the notion of task graphs and task dependences. Data-flow models are also considered closely related to functional languages. Scheduling of static task graphs on multiprocessors is one of the most widely studied topics in computer science, and the early work has been summarized in an excellent survey [Kwok and Ahmad 1999]. Recent interest in task-graph- or data-flow-like models for parallel computing is evident in projects such as CnC [Budimlić et al. 2010], OmpSs [Duran et al. 2011] and its variants, and others [Matsakis 2012; Broquedis et al. 2012; Pratikakis et al. 2011]. In the meantime, scheduling of dynamic task graphs has been an active topic [Agrawal et al. 2010; Tasirlar and Sarkar 2011].

Schedulers such as NABBIT [Agrawal et al. 2010], QUARK [YarKhan et al. 2011], StarPU [Augonnet et al. 2011], OmpSs [Duran et al. 2011], and XKaapi [Gautier et al. 2013] require the user to manage memory while specifying inter-task dependences and, possibly, data access modes. These models express data-flow dependences without building on the static single-assignment form. We consider a different programming model that schedules task graphs in single-assignment form, similar to CnC.

Memory optimization for data-flow(-like) programming systems is a well-studied problem. To the best of our knowledge, it was first studied as the problem of eliminating array copies (formally referred to as the aggregate update problem) by Hudak and Bloss [Hudak and Bloss 1985]. As summarized by Gérard et al. [2012], the solutions can be of three types. The first group involves reference counts, an approach that has been used with CnC [Budimlić et al. 2010], OpenStream [Pop and Cohen 2013], and other dynamic task scheduling [Kurt et al. 2014] strategies, but it can limit the types of programs that can be expressed and otherwise puts a large burden on the 
programmer. Other approaches include compile-time solutions [Schnorf et al. 1993; Gérard et al. 2012], which are not applicable when the task graph is dynamically generated, and solutions based on typing the operators, which are not applicable in our case (or would require an additional burden on the programmer if added to the programming model). Another recent effort with a similar focus is from Abu-Mahmeed et al. [2009] and involves scheduling to avoid unnecessary copies. Again, the work cannot support a programming model where the task graph is dynamically generated. XKaapi [Gautier et al. 2013] starts with a data-flow graph with user-specified data reuse, but it dynamically copies the data blocks to eliminate false dependences resulting from such user specification.

The issue of memory reuse in dynamic, task-graph-based systems has received limited attention. One proposal involves user-provided folding functions (similar to our recycling functions) for $\mathrm{CnC}$ [Sbirlea et al. 2012]. This work requires the user to explicitly map the data items (or single-assignment tags) to physical memory, guaranteeing a memory bound. However, folding functions for different memory bounds could be completely different, imposing an additional burden on the user to pick reasonable bounds and corresponding folding functions. We believe this was the motivation for a more recent solution involving an inspector-executor-like approach [Sbirlea et al. 2014] that explicitly expands the full task graph and determines a schedule satisfying a memory bound. In addition to explicit expansion of the task graph, this approach requires expensive analysis for each problem size. In comparison, we can derive recycling functions using execution on a smaller problem size and can efficiently detect (and recover from) an incorrect recycling function when using them in larger-scale runs.

\section{CONCLUSIONS}

Memory-efficient scheduling of single-assignment task graphs combines the simplicity of task graph specification with the practical usefulness of handling large problem sizes. We have presented a novel approach for optimizing memory usage when dynamically scheduling single-assignment task graphs. The practical approach combines user assistance in the understanding of the task graph's structure, evaluation of various recycling strategies, and dynamic recovery from incorrect recycling choices. The approach allows a user to iteratively explore the space of recycling functions, incrementally optimizing the implementation while retaining performance portability. We believe the presented approach would be ideal as a component of the tuning language employed in prevalent dynamic task graph schedulers.

Certain task graphs (e.g., fast Fourier transform) might not lend themselves to valid recycling functions. In particular, nodes in the task graph might lack other nodes that succeed all of their predecessors (Constraint 1). In these cases, our approach will detect the absence of such recycling functions, allowing the user to employ other strategies.

Throughout the article, we have focused our discussion on task graphs where all tasks produce data blocks of the same size. This allows the output produced by a task $t$ 's recycle successor to be stored in the memory region allocated to hold the data produced by $t$. In a broader sense, a task $t$ 's recycle successor identifies the point in the execution at which the data block allocated by $t$ can be safely deallocated. Therefore, our store recycling approach is also useful as a form of automated garbage collection in managing memory for task graphs producing tasks that generate data blocks of dissimilar sizes.

We have focused on computing recycling functions as a combination of true dependences in the application so that no additional dependences are introduced in the task graph, adversely affecting the critical path length or introducing cycles. In certain cases, such as FW, the most memory-efficient schedules might require introduction of additional dependences. Adding edges in the task graph to ensure validity of recycling 
functions without introducing cycles in the task graph, which can lead to deadlocks, is a non-trivial challenge. We plan to pursue this as future research.

In addition to improving memory consumption by identifying data blocks past their last use, recycling functions provide information on when a data block can be garbage collected. For future research, we plan to use this information to inform the scheduler and to construct memory-efficient schedules.

\section{REFERENCES}

Samah Abu-Mahmeed, Cheryl McCosh, Zoran Budimlić, Ken Kennedy, Kaushik Ravindran, Kevin Hogan, Paul Austin, Steve Rogers, and Jacob Kornerup. 2009. Scheduling tasks to maximize usage of aggregate variables in place. In CC. 204-219.

Kunal Agrawal, Charles E. Leiserson, and Jim Sukha. 2010. Executing task graphs using work-stealing. In Proceedings of the IEEE 24th International Symposium on Parallel \& Distributed Processing (IPDPS). $1-12$.

Arvind. 1981. Data flow languages and architecture. In Proceedings of the 8th Annual Symposium on Computer Architecture (ISCA' 81). 1.

Cédric Augonnet, Samuel Thibault, Raymond Namyst, and Pierre-André Wacrenier. 2011. StarPU: A unified platform for task scheduling on heterogeneous multicore architectures. Concurrency and Computation: Practice and Experience 23, 2 (2011), 187-198.

François Broquedis, Thierry Gautier, and Vincent Danjean. 2012. LIBKOMP, an efficient openMP runtime system for both fork-join and data flow paradigms. In Proceedings of the 8th International Conference on OpenMP in a Heterogeneous World (IWOMP'12). Springer-Verlag, Berlin, 102-115. DOI:http://dx. doi.org/10.1007/978-3-642-30961-8_8

Zoran Budimlić, Michael Burke, Vincent Cavé, Kathleen Knobe, Geoff Lowney, and others. 2010. Concurrent collections. Scientific Programming 18, 3 (2010), 203-217.

Zoran Budimlic, Aparna M. Chandramowlishwaran, Kathleen Knobe, Geoff N. Lowney, Vivek Sarkar, and Leo Treggiari. 2008. Declarative aspects of memory management in the concurrent collections parallel programming model. In Proceedings of the Workshop on Declarative Aspects of Multicore Programming. 47-58. DOI : http://dx.doi.org/10.1145/1481839.1481846

A. Bui, Kwang-Ting Cheng, J. Cong, L. Vese, Yi-Chu Wang, Bo Yuan, and Yi Zou. 2012. Platform characterization for domain-specific computing. In ASP-DAC. 94-99.

Shuai Che, Michael Boyer, Jiayuan Meng, David Tarjan, Jeremy W. Sheaffer, Sang-Ha Lee, and Kevin Skadron. 2009. Rodinia: A benchmark suite for heterogeneous computing. In Proceedings of the IEEE International Symposium on Workload Characterization (IISWC'09). IEEE, 44-54.

Jack B. Dennis. 1974. First version of a data flow procedure language. In Programming Symposium. Springer, 362-376.

Alejandro Duran, Eduard Ayguadé, Rosa M. Badia, Jesús Labarta, Luis Martinell, Xavier Martorell, and Judit Planas. 2011. OmpSs: A Proposal for Programming Heterogeneous Multi-core Architectures. Parallel Processing Letters 21, 2 (2011), 173-193.

Thierry Gautier, Joao V. F. Lima, Nicolas Maillard, and Bruno Raffin. 2013. XKaapi: A runtime system for data-flow task programming on heterogeneous architectures. In Proceedings of the 2013 IEEE 27th International Symposium on Parallel \& Distributed Processing (IPDPS). IEEE, 1299-1308.

Léonard Gérard, Adrien Guatto, Cédric Pasteur, and Marc Pouzet. 2012. A modular memory optimization for synchronous data-flow languages: Application to arrays in a lustre compiler. SIGPLAN Not. 47, 5 (June 2012), 51-60.

Paul Hudak and Adrienne G. Bloss. 1985. The aggregate update problem in functional programming systems. In POPL. 300-314.

Prabhanjan Kambadur, Anshul Gupta, Torsten Hoefler, and Andrew Lumsdaine. 2009. Demand-driven execution of static directed acyclic graphs using task parallelism. In Proceedings of the 2009 International Conference on High Performance Computing (HiPC). IEEE, 284-293.

Mehmet Can Kurt, Sriram Krishnamoorthy, Kunal Agrawal, and Gagan Agrawal. 2014. Fault-tolerant dynamic task graph scheduling. In SC. 719-730.

Yu-Kwong Kwok and Ishfaq Ahmad. 1999. Static scheduling algorithms for allocating directed task graphs to multiprocessors. Comput. Surveys 31, 4 (Dec. 1999), 406-471.

Malcolm Yoke Hean Low, Weiguo Liu, and Bertil Schmidt. 2007. A parallel BSP algorithm for irregular dynamic programming. In APPT. 151-160.

Nicholas D. Matsakis. 2012. Parallel closures: A new twist on an old idea. In HotPar. 5-5. 
Walid A. Najjar, Edward A. Lee, and Guang R. Gao. 1999. Advances in the dataflow computational model. Parallel Comput. 25, 1314 (1999), 1907-1929.

Antoniu Pop and Albert Cohen. 2013. OpenStream: Expressiveness and data-flow compilation of OpenMP streaming programs. ACM Transactions on Architecture and Code Optimization (TACO) 9, 4 (2013), 53.

Polyvios Pratikakis, Hans Vandierendonck, Spyros Lyberis, and Dimitrios S. Nikolopoulos. 2011. A programming model for deterministic task parallelism. In Proceedings of the 2011 ACM SIGPLAN Workshop on Memory Systems Performance and Correctness. ACM, 7-12.

Veselin Raychev, Martin Vechev, and Manu Sridharan. 2013. Effective race detection for event-driven programs. In OOPSLA. 151-166. DOI : http://dx.doi.org/10.1145/2509136.2509538

Dragos Sbirlea, Zoran Budimlic, and Vivek Sarkar. 2014. Bounded memory scheduling of dynamic task graphs. In PACT. 343-356. DOI : http://dx.doi.org/10.1145/2628071.2628090

Dragos Sbirlea, Kathleen Knobe, and Vivek Sarkar. 2012. Folding of tagged single assignment values for memory-efficient parallelism. In Euro-Par. 601-613.

Peter Schnorf, Mahadevan Ganapathi, and John L. Hennessy. 1993. Compile-time copy elimination. Software: Practice and Experience 23, 11 (1993), 1175-1200.

Sagnak Tasirlar and Vivek Sarkar. 2011. Data-driven tasks and their implementation. In ICPP. $652-661$. DOI : http://dx.doi.org/10.1109/ICPP.2011.87

Asim YarKhan, Jakub Kurzak, and Jack Dongarra. 2011. Quark users guide: Queueing and runtime for kernels. University of Tennessee Innovative Computing Laboratory Technical Report ICL-UT-11-02.

Received May 2016; revised November 2016; accepted November 2016 